\title{
Enhanced MRI-guided radiotherapy with gadolinium-based nanoparticles: preclinical evaluation with an MRI-linac
}

\author{
H. L. Byrne $1^{*} \mathbb{D}$, G. Le Duc ${ }^{2}$, F. Lux ${ }^{2,3,4}$, O. Tillement ${ }^{2,3}$, N. M. Holmes ${ }^{5}$, A. James ${ }^{6}$, U. Jelen ${ }^{6}$, B. Dong ${ }^{6}$, G. Liney ${ }^{6}$, \\ T. L. Roberts ${ }^{6,7}$ and Z. Kuncic ${ }^{1}$
}

${ }^{*}$ Correspondence:

hilary.byrne@sydney.edu.au

${ }^{1}$ Institute of Medical Physics, School of Physics, The

University of Sydney, Sydney, NSW 2006, Australia

Full list of author information is available at the end of the article

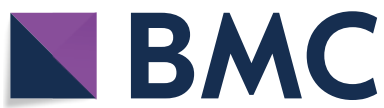

(0) The Author(s) 2020. This article is licensed under a Creative Commons Attribution 4.0 International License, which permits use, sharing, adaptation, distribution and reproduction in any medium or format, as long as you give appropriate credit to the original author(s) and the source, provide a link to the Creative Commons licence, and indicate if changes were made. The images or other third party material in this article are included in the article's Creative Commons licence, unless indicated otherwise in a credit line to the material. If material is not included in the article's Creative Commons licence and your intended use is not permitted by statutory regulation or exceeds the permitted use, you will need to obtain permission directly from the copyright holder. To view a copy of this licence, visit http://creativecommons.org/ licenses/by/4.0/. The Creative Commons Public Domain Dedication waiver (http://creativecommons.org/publicdomain/zero/1.0/) applies to the data made available in this article, unless otherwise stated in a credit line to the data.
Methods: Treatments were carried out on F344 Fischer rats bearing a $9 \mathrm{~L}$ glioma brain tumour. Animals received either: (A) no treatment; (B) injection of nanoparticles followed by $M R I ;(C)$ radiotherapy with $M R I$; or $(D)$ injection of nanoparticles followed by radiotherapy with MRI. Pre-clinical irradiations were carried out on the 1.0 T, $6 \mathrm{MV}$ inline Australian MRI-linac. Imaging used a custom head coil specially designed to minimise interference from the radiotherapy beam. Anaesthetised rats were not restrained during treatment but were monitored with a cine-MRI sequence. Inductively Coupled Plasma Mass Spectrometry (ICP-MS) analysis was used to quantify residual gadolinium in the brain in normal and tumour tissue.

Results: A preclinical evaluation of nano-enhanced radiation treatment has been carried out on a 1.0 T MRI-linac, establishing a workflow on these novel systems. Extension of life when combining radiotherapy with nanoparticles was not statistically different from that for rats receiving radiotherapy only. However, there was no detrimental effect for animals receiving nanoparticles and radiation treatment in the magnetic field compared with control branches. Cine-MR imaging was sufficient to carry out monitoring of anaesthetised animals during treatment. AGuIX nanoparticles demonstrated good positive contrast on the MRI-linac system allowing confirmation of tumour extent and nanoparticle uptake at the time of treatment.

Conclusions: Novel nano-enhanced radiotherapy with gadolinium-containing nanoparticles is ideally suited for implementation on an MRI-linac, allowing a workflow 
with time-of-treatment imaging. Live irradiations using this treatment workflow, carried out for the first time at the Australian MRI-linac, confirm the safety and feasibility of performing MRI-guided radiotherapy with $A G u I{ }^{\circledR}$ nanoparticles. Follow-up studies are needed to demonstrate on an MRI-linac the radiation enhancement effects previously shown with conventional radiotherapy.

\section{Background}

Many technological developments in radiation oncology focus on integrating existing treatment and imaging modalities. Exercising the two modalities concurrently eliminates or reduces registration errors and temporal variations and opens new avenues of investigation and improvement. The emergence of the MRI-linac, a single system incorporating an MRI (magnetic resonance imaging) scanner with a linear accelerator (linac), has given unprecedented insight into inter- and intra-fraction movement of soft tissue targets, enabling adaptive radiotherapy strategies (Acharya et al. 2016; Winkel et al. 2019).

There are currently four types of MRI-linac systems worldwide (Liney et al. 2018b) with increasing adoption in the clinic of the two leading commercial offerings: the ViewRay MRIdian with a $0.35 \mathrm{~T}$ magnet (Klüter 2019) and the Elekta Unity with a $1.5 \mathrm{~T}$ magnet (Raaymakers et al. 2017). Both systems introduce the patient parallel to the magnetic field direction along the bore of the magnet, with a split magnet design leaving a gap for the radiotherapy beam to be delivered perpendicular to the magnetic field. There are two alternative research-only systems which are capable of introducing the patient at right angles to the magnetic field allowing the radiotherapy beam to be delivered colinearly to the magnetic field, down the bore of the magnet. These are the Australian MRIlinac (Keall et al. 2014) and the Aurora-RT from MagnetTx (Fallone 2014).

The primary clinical interest in MRI-linacs has hinged on the expectation of adapting treatment both inter- and intra-fraction, though much work is still to be done on realising efficient workflows and prioritising the targets with most to gain from motion adaptation (Corradini et al. 2019). Fundamentally, these innovations strive to make existing radiotherapy techniques ever more precise and ever more conformal to the target. Hence, they could be considered improvements to existing techniques rather than intrinsically novel treatment approaches.

In contrast, nano-enhanced radiotherapy (or nanoparticle radio-enhancement) is an emerging field in radiotherapy where it is proposed that the fundamental physical and biological methods of cell kill can be modulated locally within the tumour to give therapeutic advantage (Kuncic and Lacombe 2018; Byrne et al. 2020). Many in vitro and in vivo studies have shown that radiobiological damage (McMahon et al. 2011; Porcel et al. 2012) and tumour radiation response (Bobyk et al. 2013; Hainfeld et al. 2013) are enhanced by the presence of high-atomic number nanoparticles. A body of theoretical and Monte Carlo simulation research has identified changes in the patterns of radiation damage due to the presence of nanoparticles which may give rise to the enhanced biological effect (Byrne et al. 2019; Butterworth et al. 2012; Lechtman et al. 2013; McNamara et al. 2016; Brown et al. 2018).

The nanoparticle $\mathrm{AGuIX}^{\circledR}$ (NH TherAguix, France), consisting of a polysiloxane core surrounded by chelated gadolinium atoms, has been designed to enhance both 
radiotherapy treatment and MRI contrast (Detappe et al. 2015). Many preclinical studies have been carried out using the European synchrotron, preclinical kilovoltage radiation sources and clinical 6 MV beams (Le Duc et al. 2011; Dufort et al. 2015; Verry et al. 2016; Lux et al. 2018). These studies have demonstrated that, in preclinical models, the presence of $\mathrm{AGuIX}^{\circledR}$ nanoparticles within the tumour during radiotherapy boosts the efficacy of radiotherapy leading to longer survival compared to radiotherapy treatment alone.

The first-in-human injection of AGuIX ${ }^{\circledR}$ was successfully performed at Grenoble hospital in July 2016 within the phase I NANORAD clinical trial [NCT02820454] (Verry et al. 2019; ClinicalTrials.gov) making it one of the very few nanoparticle therapies to progress to clinical translation. This clinical trial for brain metastases patients showed excellent tolerance to the delivered dose and clinical benefit for 13 out of 14 patients, leading to recruitment for Phase II of NANORAD [NCT03818386] (ClinicalTrials.gov).

As a nanoparticle with capacity for both MRI contrast and radiation enhancement, AGuIX $^{\circledR}$ is ideally suited for therapeutic use on an MRI-linac. Nanoparticle uptake is a key determinant of the efficacy of nanoparticle radiation enhancement in a clinical setting. It is to be expected that inter-patient, inter-lesion and intra-lesion variation in uptake will be seen, that this will vary over time, and that the ability of a lesion to take up the nanoparticles may be altered by treatment. An MRI-linac allows measurement of this key uptake variable at the time of treatment. The aim of the study reported here was, therefore, to validate the feasibility of carrying out MRI contrast-enhanced and nanoparticle-enhanced radiotherapy treatment on an MRI-linac. The first live irradiation on the Australian MRI-linac was carried out as part of this study, with the dosimetry and imaging sequences previously reported (Liney et al. 2019); this work reports on the survival study, nanoparticle uptake and ICP-MS results, and details the MRI-linac workflow.

\section{Results}

Tumour model

A syngeneic model of brain cancer in rats was utilised, where adult rats were injected with cancer cells via stereotaxic surgery. Tumour implantation was successful in nearly all animals with the failure of tumour growth in only 2 animals (out of 32 animals in the survival trial and 43 in pilot stages) attributed to a clogged needle preventing delivery of tumour cells. One animal was lost to brain haemorrhage 4 days after surgery. There were no complications of tumour growth outside the skull. Figure 1 shows typical tumour growth at day 11 and day 18 .

\section{MR imaging}

All animals in groups B (nanoparticles only), C (radiotherapy only) and D (nanoparticles and radiotherapy) received a T2-weighted scan to confirm positioning and a T1-weighted scan which would confirm enhanced tumour contrast if nanoparticles had been administered, before commencing cine-MRI scans for $17 \mathrm{~min}$. If the animals received radiotherapy, this was delivered concurrent to the cine-MRI. Figure 2 shows T1-weighted images taken over a 1-h period, 20-70 min post-injection of AGuIX nanoparticles, with a plot of signal intensity from a small region of interest at the tumour centre. The brain appears dark due to little transfer of contrast agent 

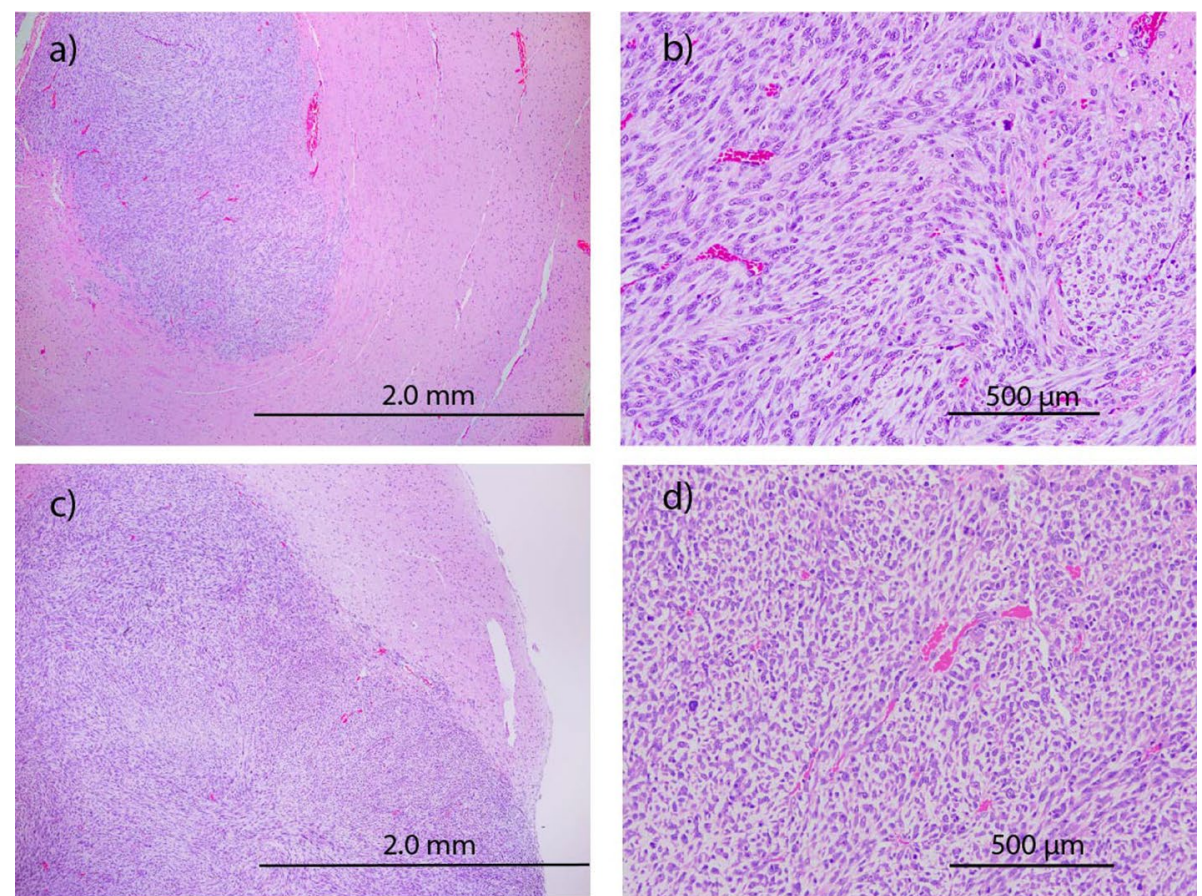

Fig. 1 Hemotoxalin and eosin staining of rat brain showing typical tumour growth at day 11: a 4x magnification; b 20x magnification and day 18: c $4 \times$ magnification; $\mathbf{d} 20 \times$ magnification

across the blood-brain barrier, while the tumour is clearly seen as an enhancing, elongated lesion in the front right hemisphere. AGuIX provided excellent contrast on the T1-weighted images and showed a slow wash-out with significant retention after $1 \mathrm{~h}$ (see Fig. 2).

Figure $1 \mathrm{~h}$ ) shows an example of the T2-weighted image used to confirm positioning after aligning the animal on the couch with the in-room lasers. The images in Fig. 1i, $j$ are examples of still images from the cine-MRI sequence taken to monitor the animals during radiotherapy treatment. No change in image quality was observed when the beam was on compared to when it was off. Diaphragm movement and expansion of the lungs were clearly visible (see additional movie file "Additional file 1" showing the real-time imaging - the radiation beam was turned on 50 frames into the video, with signal strength and signal-noise ratio unaffected, as previously reported in Liney et al. (2019)).

\section{ICP-MS}

Results of ICP-MS analysis of brain tissue from animals given AGuIX injection are shown in Fig. 3. The replicates are from separate animals collected following end-stage euthanasia-three from group B (nanoparticles only) at 8, 9 and 10 days post-treatment and three from group D (nanoparticles and radiotherapy) at 11, 10 and 10 days posttreatment. Unfortunately, one tumour tissue sample from group B was contaminated during preparation. Analysis of the normal brain tissue yielded gadolinium content between $4.86-16.55 \mu \mathrm{g} / \mathrm{kg}$, up to one order of magnitude below the content in tumour 

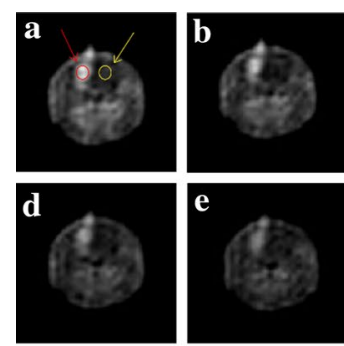

$\mathbf{e}$
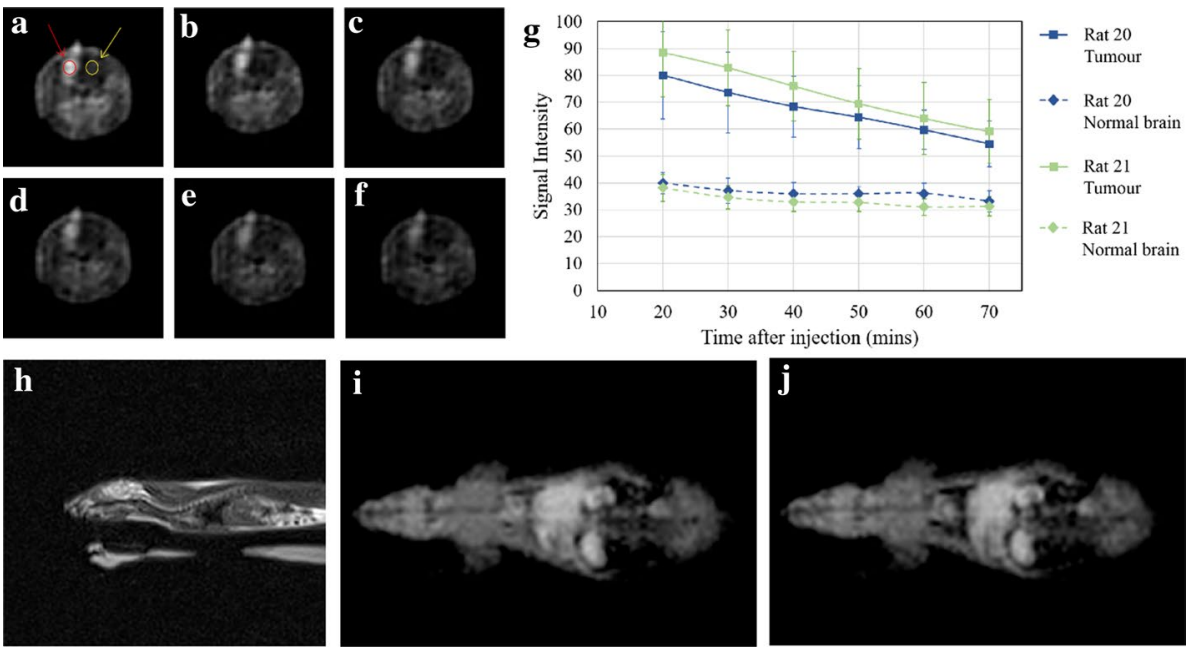

Fig. 2 a-fT1-weighted MRI images in the axial plane showing day 14 tumour enhancement following injection of AGulX, taken at 10-min time intervals between a 20 min- $\mathbf{f} 70$ min post-injection. $\mathbf{g}$ Plot of the average $\mathrm{T1}$-weighted signal intensity with standard deviation in the regions of interest indicated in a for tumour (red) and normal brain (yellow). $\mathbf{h}$ Representative T2-weighted sagittal image showing brain for positioning. i full-exhale frame and $\mathbf{j}$ full-inhale frame taken from cine-MRI acquired in the coronal plane

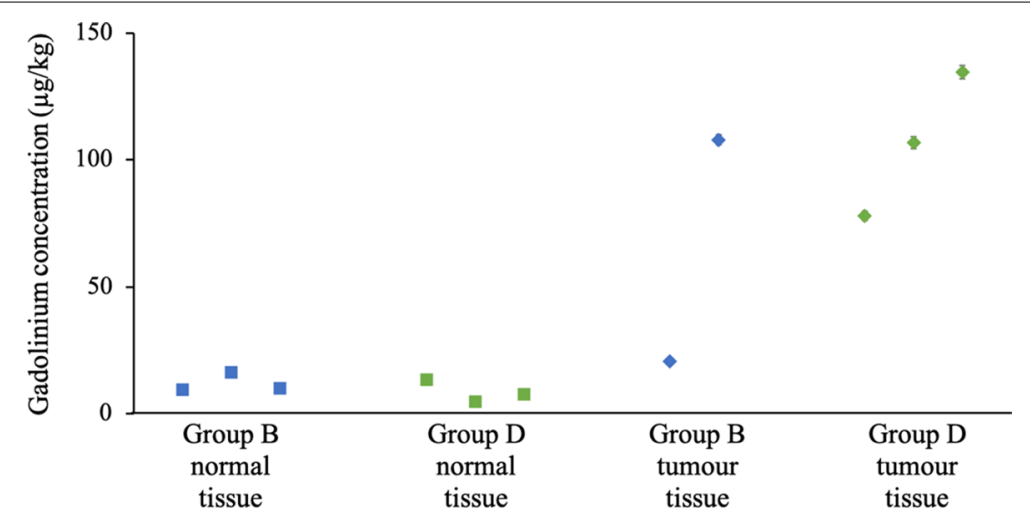

Fig. 3 Gadolinium content of brain tissue from ICP-MS analysis. Tissue was taken from the tumour and normal brain tissue of $n=3$ animals in group $B$ (nanoparticles only) and $n=3$ in group $D$ (nanoparticles and radiotherapy) following end-stage euthanasia. One sample of tumour tissue in group B was lost during preparation for ICP-MS analysis

tissue. For animals that did not receive an injection of nanoparticles, group A (no treatment) and group C (radiotherapy only), samples were similarly collected. Gadolinium content in both tumour and normal tissue was below detectable limits (results not shown).

\section{Survival data}

Figure 4 shows the Kaplan-Meier survival curves for all treatment groups. The mean survival and standard deviation for group A was $20.14 \pm 1.68$ days and for 
group B was $20.29 \pm 1.50$ days. Group C animals showed a short extension of life to $22.56 \pm 1.67$ days. Using the log-rank test (Graphpad Prism 8.2.1), this is a statistically significant extension compared to the control branch A $(p=0.0096)$. Animals in group D receiving nanoparticles and radiotherapy showed median survival of $22.44 \pm 1.51$ days. Again, with the log-rank test, this is statistically significant compared to the control branch ( $p=0.0095)$, but not statistically different from group $C$.

\section{Discussion}

This study has carried out the first proof of concept of MRI-guided nanoparticle radioenhancement on an MRI-linac. The results demonstrate that the technique is safe to deliver in the presence of a magnetic field with the administration of nanoparticles combined with MRI causing no significant change to survival post-treatment. This study also represents the first preclinical study on an MRI-linac, machines which are currently only developed for human treatment, identifying a workflow for future preclinical work supporting novel treatment development.

As mentioned in the introduction, the AGuIX nanoparticle has been shown to enhance radiotherapy in many previous studies (Le Duc et al. 2011; Dufort et al. 2015; Verry et al. 2016). However, in those studies and in the Phase I NANORAD human clinical trial, the MRI images were necessarily taken on a separate machine at times several hours apart from the delivery of radiotherapy. In this study, MR imaging at time of treatment was used to confirm the presence of a tumour and verify that successful administration of nanoparticles with uptake to the tumour was achieved without the need for a separate scan requiring additional anaesthesia for the animals. In future clinical trials, radiotherapy on an MRI-linac with an appropriate imaging protocol for T1 mapping will allow quantification of the uptake of nanoparticles in the tumour at the actual time of treatment with each fraction delivered. This more accurate measure of the heterogeneous and patient-specific uptake should give superior confidence in the analysis correlating patient response to nanoparticle uptake and potentially allow adaptation of radiation dose to compensate for differing uptake.

The primary limitation to this study is that the extension of life achieved with a single delivery of 10 Gy at day 11 was limited. It is likely that a modest but clinically significant percentage increase in lifespan caused by nanoparticle enhancement was not observed with this protocol due to the aggressive nature of the tumour. The decision was taken not to escalate the radiation dose further out of concern for increased possibility of normal tissue complication and the animals' tolerance of increased cranial inflammation in addition to the existing tumour burden and effect of the ketamine/xylazine anaesthesia. We recommend that future studies involving animals at the Australian MRI-linac should install access to isoflurane anaesthesia in the linac bunker. This mode of anaesthesia does not cause as much stress for the animals as the ketamine/xylazine, allowing radiation dose escalation or multiple fractions to be delivered. In future studies, it is of interest to investigate the effect of varying the concentration of AGuIX administered on the tumour uptake and synergistic effects with radiotherapy dose.

Cine-MRI proved a useful tool for monitoring the animals during radiation delivery (see Additional file 1). The frame rate ( 2 frames per second) was sufficient to note diaphragm motion and confirm the presence of breathing, though with an average 
breathing rate of 1 breath per second visualisation did not capture the full diaphragm motion. The cine-MRI also served to confirm that the animal remained in positionalthough anaesthetised, animals may still twitch or move out of position. Any significant head movement displaces the target out of the beam and brings normal tissue into the field, potentially causing detrimental effects for the animal. Cine-MRI was sufficient to allow movement to be immediately observed and in one test case, the beam could be manually shut-off within less than $2 \mathrm{~s}$.

The MR images provided by the unique radiation-beam-compatible head coil maintained image quality during radiation delivery with no increase in image noise. The 1.3$\mathrm{mm}$ in-plane resolution was sufficient for confirming beam targeting and the presence of a tumour, however, was not a high enough resolution to allow quantification of uptake heterogeneity across the tumour. As the head coil used was large compared to the size of the animal, the signal-noise ratio limited the frame rate that could be achieved for cine-MRI. Future work could include the design of a smaller, higher-resolution RF coil for dedicated preclinical use on the MRI-linac that would further improve signal-noise ratio and facilitate quantitation of the nanoparticle uptake.

While gadolinium provides excellent MRI contrast, gadolinium retention following administration of multiple doses in patients with compromised kidney function has been linked to nephrogenic systemic fibrosis (Center for Drug Evaluation and Research 2019). Macro-cyclic chelating structures such as that in currently approved MRI contrast agents and AGuIX are more robust than older linear molecules, but retention should still be studied to ensure safety. Our ICP-MS analysis of rat brain tissue 8-11 days following AGuIX injection shows that gadolinium is retained in tumour tissue in the brains of animals who received a single injection of AGuIX. Small amounts of gadolinium are also seen in the normal brain tissue, but without further investigation, we cannot say whether this is due to extravasation in the tissue or residual amounts in the circulating blood. However, the amounts retained are comparable to findings in human brain tissue over a similar time-frame following administration of current FDA-approved MRI contrast agents, for example Kiviniemi et al. (Kiviniemi et al. 2019) find 0-403 $\mu \mathrm{g} / \mathrm{kg}$ in normal brain tissue and $0-1648 \mu \mathrm{g} / \mathrm{kg}$ in glioma.

$\mathrm{NH}$ TherAguix has carried out the NANORAD Phase I human clinical trial for brain metastases patients in France and are currently recruiting for a Phase II trial (NCT03818386). However, a human clinical trial of nanoparticle-enhanced radiotherapy on an MRI-linac would provide fraction-by-fraction uptake data with no inconvenience to the patient. This would more accurately quantify nanoparticle penetration in the tumour, and the variability in uptake both inter- and intra-patient and inter-fraction, allowing a more stringent analysis of the radio-enhancement effect. If the biological effect dependent on uptake can be rigorously modelled, this would allow adaptive treatment delivery to compensate for patient-specific uptake and tumour inhomogeneity.

\section{Conclusions}

Nano-enhanced radiotherapy using a gadolinium-based nanoparticle such as AGuIX is ideally suited for implementation on new MRI-linac systems due to the superior accuracy of determining nanoparticle uptake at time of treatment. A preclinical study has 
been carried out on the Australian MRI-linac representing the first in vivo nanoparticle radio-enhancement experiment carried out in the presence of a high (1 T) magnetic field and including the first live irradiation at the research facility. Delivery of a single radiotherapy dose at day 11 (10 Gy to whole brain) gave a modest extension in mean survival from 20.1 to 22.6 days in a very aggressive tumour model. Injection of gadolinium-based nanoparticles combined with radiotherapy treatment in the magnetic field showed no statistically significant change in mean survival.

Time-of-treatment imaging was used to confirm the existence of a tumour showing enhanced T1-weighted MRI contrast due to nanoparticle uptake before treatment commenced. Cine-MRI enabled monitoring of the anaesthetised animals during radiotherapy delivery, removing the necessity to restrain the animals. Imaging performance was maintained during treatment delivery with no visible effect from radiation induced noise.

This study demonstrates a workflow for preclinical evaluation of novel nanotheranostics on recently developed MRI-linac systems.

\section{Methods}

A 4-branch survival study was carried out using adult male Fischer rats implanted with GS-9L glioma cells. Animals received either: A) no treatment; B) injection of nanoparticles with MRI; C) radiotherapy with MRI; D) injection of nanoparticles followed by radiotherapy with MRI. Figure 5 shows a schematic of the protocol. Radiotherapy was delivered in a single $10 \mathrm{~Gy}$ fraction on the Australian MRI-linac.

All animal experiments were approved by the Animal Care and Ethics committee of Western Sydney University (ACEC number A12431) and were conducted in accordance with the "Australian Code for the Care and Use of Animals for Scientific Purposes (2013)" and the "New South Wales Animal Research Act 1985".

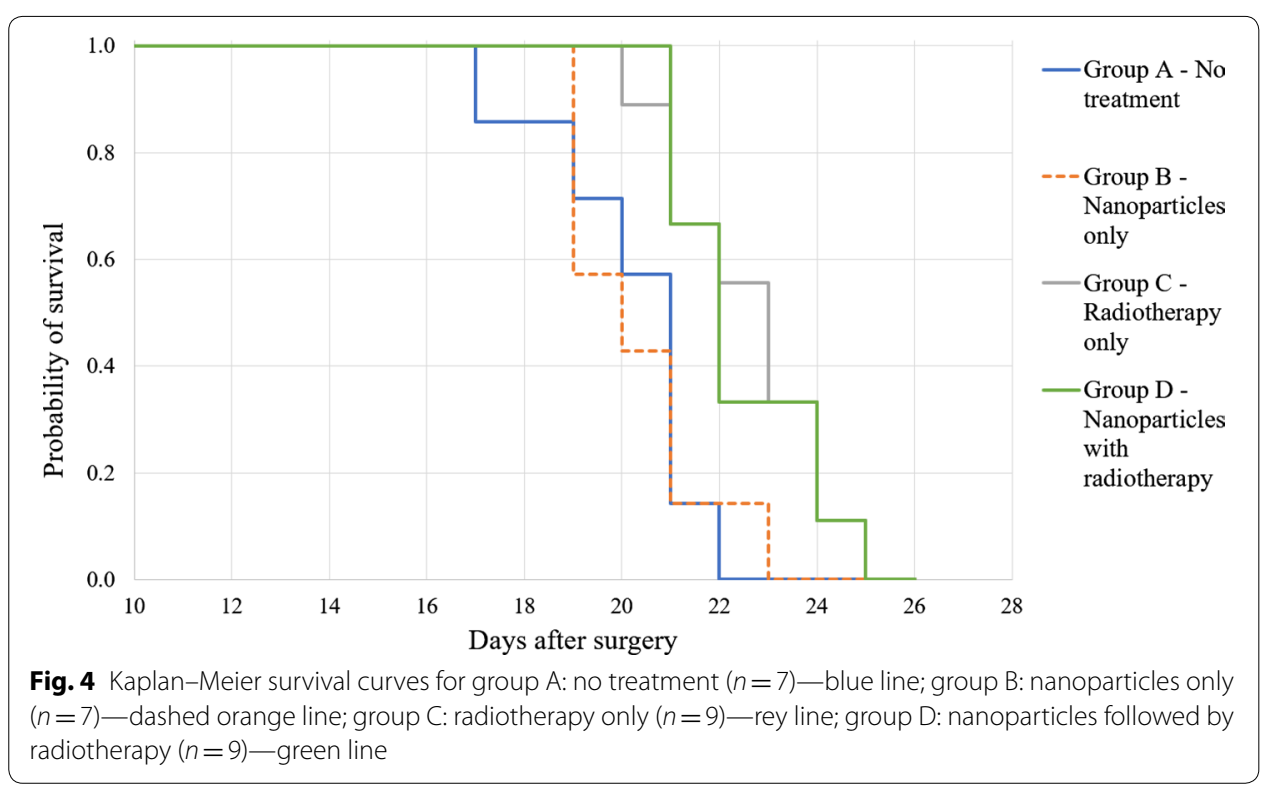




\section{Cell culture}

GS-9L glioma cells (CellBank Australia ECACC 94110705) (Schmidek et al. 1971) were cultivated in EMEM $+2 \mathrm{mM}$ Glutamine+1\% Non-Essential Amino Acids $($ NEAA $)+10 \%$ Foetal Bovine Serum following the suppliers instructions. Harvested cells for implantation were confirmed to be more than $90 \%$ viable by trypan blue exclusion staining.

\section{Tumour implantation}

Surgery was carried out on day 0. Adult Fischer F344 rats were operated on at 13-15 weeks of age (weight range 250-300 g). As described in Verry et al. (Verry et al. 2016), using a Kopf stereotaxic frame and a Hamilton microlitre syringe, $10^{4}$ GS-9L glioma cells suspended in $1 \mu \mathrm{L}$ of serum-free cell growth medium were implanted to the right caudate nucleus, at $3.5 \mathrm{~mm}$ lateral to bregma and $6 \mathrm{~mm}$ depth. The injection was carried out slowly using the Kopf micro-injection unit (>30 s to deliver $1 \mu \mathrm{L}$ ) and the needle allowed to dwell for 5 min before slow retraction. The hole in the skull was plugged with bone wax to prevent tumour cell growth above the skull before suturing the surgical wound. A local anaesthetic, bupivacaine, was injected under the skin prior to the first incision over the skull, and carprofen was administered as pain relief during the surgery. Animals were monitored at least once daily following surgery until euthanasia.

\section{Histology}

Haematoxylin and eosin staining of brain tumour samples at different stages of growth was carried out to confirm tumour morphology.

\section{AGuIX nanoparticles}

Nanoparticles were supplied by NH TherAguix in dry powder form in sterile vials. The nanoparticles have been previously described and characterised (Mignot et al. 2013; Lux et al. 2018; Verry et al. 2020). In brief, the nanoparticle consists of a polysiloxane core surrounded by covalently grafted cyclic ligands of gadolinium, derived from DOTA (1,4,7,10-tetraazacyclododecane-1,4,7,10-tetraacetic acid). The hydrodynamic diameter is $3 \pm 1.5 \mathrm{~nm}$ and the mass $10 \pm 5 \mathrm{kDa}$. The nanoparticle has a zeta potential of $10 \mathrm{mV}$ at $\mathrm{pH}$ 7.5. The biodistribution and pharmacokinetics of AGuIX have been previously investigated in several publications as summarised in Lux et al. (2018). Nanoparticles were injected intravenously via the tail vein $(100 \mathrm{mg}$ in $1 \mathrm{~mL}$ sterile water, i.e., $100 \mathrm{mM}$ $\left.[\mathrm{Gd}]_{\mathrm{eq}}\right)$.

\section{Treatment protocol}

Animals were treated on day 11 when the tumour was sufficiently vascularised to give good uptake of the nanoparticle as verified by T1-weighted image enhancement in an MR imaging pilot study. Four treatment branches were pursued as described above (see Fig. 5).

All animals were anaesthetised with isoflurane gas (induction 3\%, maintenance $2-2.5 \%)$. Groups B and D received intravenous injection of $\mathrm{AGuIX}^{\circledR}$ nanoparticles via 


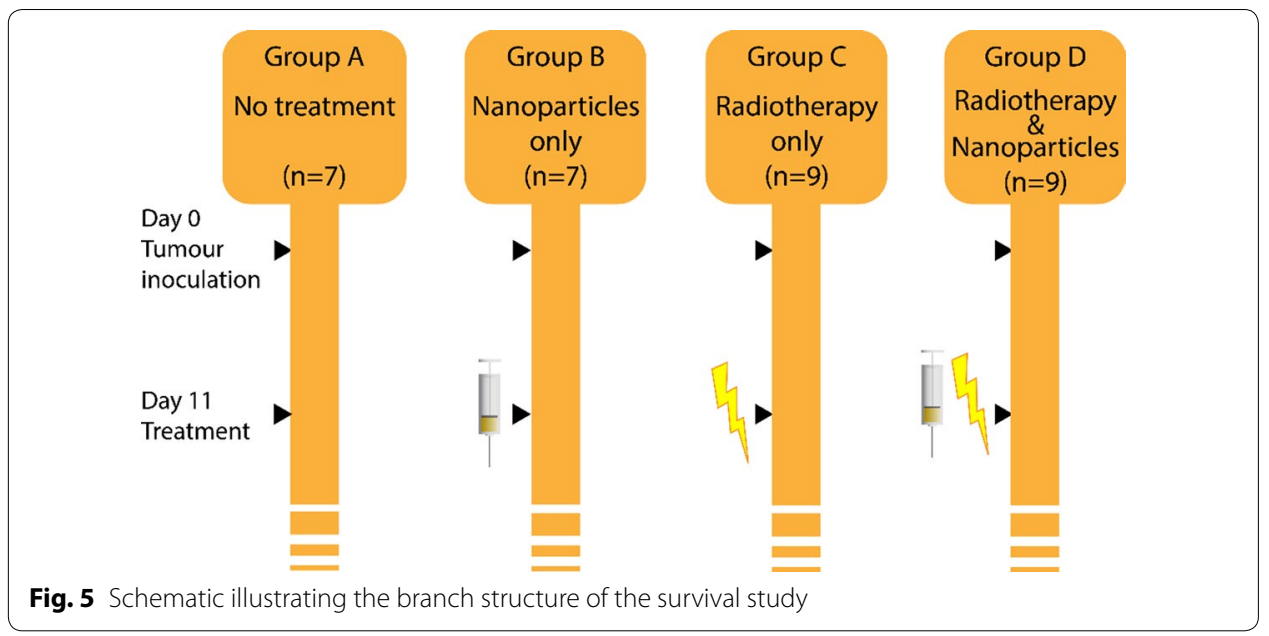

the tail vein (100 mg in $1 \mathrm{~mL}$ sterile water, i.e.. $\left.100 \mathrm{mM}[\mathrm{Gd}]_{\mathrm{eq}}\right)$. Groups A and C were kept under anaesthetic for a representative $5 \mathrm{~min}$ but did not receive an injection.

Ketamine/xylazine anaesthetic was administered intraperitoneally at an initial dosage of $72 \mathrm{mg} / \mathrm{kg}$ ketamine $/ 6 \mathrm{mg} / \mathrm{kg}$ xylazine $/ 6 \mathrm{ml} / \mathrm{kg}$ saline. If anaesthesia was not achieved after $5 \mathrm{~min}$, a top-up was administered at a dosage of $6 \mathrm{mg} / \mathrm{kg}$ ketamine $/ 0.5 \mathrm{mg} / \mathrm{kg}$ xylazine $/ 0.5 \mathrm{ml} / \mathrm{kg}$ saline, with up to 3 top-ups given in total. This produced deep anaesthesia for the minimum $1 \mathrm{~h}$ required for treatment.

Once anaesthesia was established, animals in groups B, C and D were transported from the biological resources unit to the MRI-linac bunker and positioned on the treatment couch. Body temperature was maintained by placing the animal on a towelwrapped microwaveable gel heat pad. Imaging and radiotherapy protocol is described below, in brief: localiser, T2 and T1 scans were carried out followed by cine-MRI. If the animals received radiotherapy, this was delivered concurrent to the cine-MRI. Group B (nanoparticles only) received the full imaging sequence including cine-MRI for the same duration as the other groups but no radiotherapy was delivered. Group A (no treatment) was not transported to the bunker but was placed on the towel-wrapped gel heat pad for the equivalent length of time corresponding to transportation and treatment in the other branches. The time elapsed between injection of AGuIX nanoparticles in the biological resources unit and delivery of radiotherapy in the MRI-linac bunker was on average 31 min (range 25-40 min dependent on time taken to establish sufficient depth of ketamine/xylazine anaesthesia).

Recovery from anaesthetic took place in the biological resources unit. Animals were given supplementary oxygen until they gained the righting reflex and were able to move around the cage. A heat pad was provided under part of the cage for the first night following treatment and soft food was provided on the floor of the cage.

At the first clinical signs of disease progression (decreased appetite and movement, hunching), animals were euthanised by intraperitoneal injection of pentobarbital sodium. Final progression of the disease is very fast; it is estimated that animals were euthanised less than $24 \mathrm{~h}$ before natural death would have occurred. 


\section{MR imaging}

MR imaging was carried out using a 6-channel receive-only RF coil specially designed to afford free passage of the radiation beam. In brief, rather than pursuing radiotranslucent RF coils, this coil utilises a physically open magnet and RF coils to avoid radiation induction effects, reduce beam scatter and allow interference-free imaging during treatment. The coil is described in detail in Liney et al. (Liney et al. 2018a). Originally designed as a head coil, the gel heat pads used to maintain the rat's body temperature provided a filling factor sufficient to allow imaging.

The following imaging sequence (see Fig. 6) was run for all animals receiving imaging, as previously described (Liney et al. 2019). Briefly, a 30-s localiser scan was acquired prior to a T2-weighted scan (turbo spin echo, TE/TR $=86 \mathrm{~ms} / 13493 \mathrm{~ms}$, slice thickness $5 \mathrm{~mm}$, in-plane resolution $1.3 \mathrm{~mm}$ ). This allowed the position of the brain to be confirmed with respect to the radiation isocentre. A T1-weighted scan (fast gradient echo, $\mathrm{TE} / \mathrm{TR}=1.4 \mathrm{~ms} / 3.75 \mathrm{~ms}$, slice thickness $1.4 \mathrm{~mm}$, resolution $1.3 \mathrm{~mm}$ ) for animals which had received an injection of nanoparticles, confirmed the presence of a tumour and uptake of nanoparticles through an enhancing spot in the front right hemisphere of the brain.

A cine-MRI sequence of images (dynamic fast gradient echo, TE/ $\mathrm{TR}=4.29 \mathrm{~ms} / 10 \mathrm{~ms}$, single $10-\mathrm{mm}$ slice, resolution $2.0 \mathrm{~mm}$, temporal resolution $2 \mathrm{~Hz}$ ) was then commenced along with radiation delivery. This allowed monitoring of the animal's position during treatment and monitoring of breathing to assess depth of anaesthesia. This was continued for the 17-min duration of the 10-Gy radiation treatment. For Group B animals not receiving radiation, the full MRI sequence including cine-MRI was still carried out for consistency.

\section{Radiotherapy}

Radiotherapy treatment was delivered to animals in groups $C$ (without nanoparticles) and $\mathrm{D}$ (with nanoparticles). The radiotherapy beam at the Australian MRI-linac is a $6 \mathrm{MV}$ Varian Linatron, with treatment quality assurance and small-field dosimetry carried out as previously described in detail in Liney et al. (Liney et al. 2019). Briefly, the variable source to iso-centre distance was set at $1.8 \mathrm{~m}$. After positioning the rat on the couch by aligning the eye and ear to the in-room lasers, a 1-cm grid aligned to isocenter was overlaid on the T2-weighted MR image to confirm that the rat was

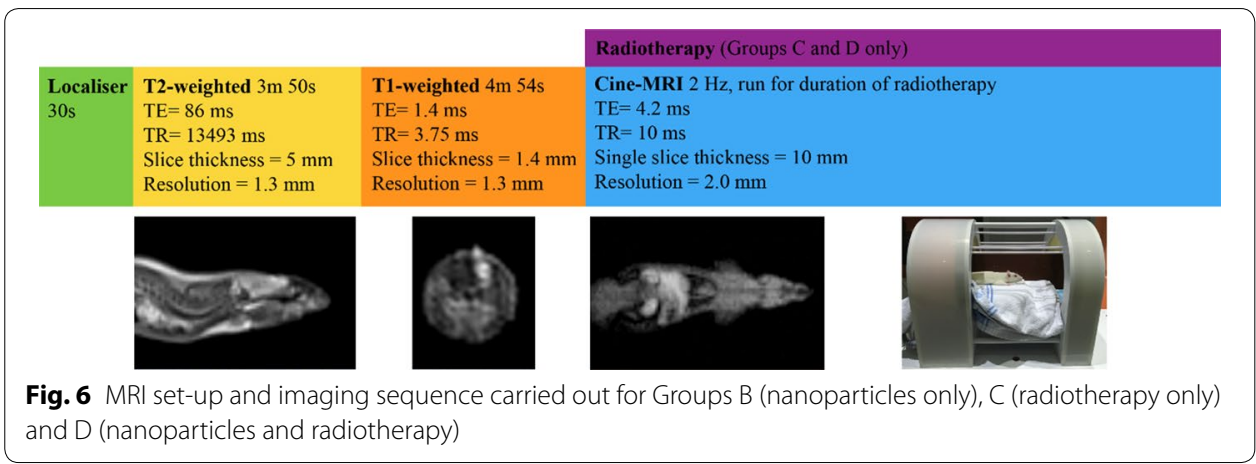


positioned correctly and the base of the field was aligned to the base of the brain. After the T1-weighted scan which would confirm enhanced tumour contrast if nanoparticles had been administered, a single dose of 10 Gy was delivered over approximately 17 min using a $2.25 \times 2.90 \mathrm{~cm}$ rectangular field covering the whole brain.

\section{ICP-MS}

Brain tissue was collected from three rats in each of the four treatment branches following end-stage euthanasia (for groups B (nanoparticles only) and D (nanoparticles and radiotherapy), this was between 8 and 11 days post-treatment) and stored in formalin. Samples of tumour and normal tissue were dissected by eye and dried for $1 \mathrm{~h} 20 \mathrm{~min}$ in a cryo-freezer until no visible liquid remained. $400 \mu \mathrm{L}$ of $70 \%$ ultra-pure nitric acid was added and the samples left until fully digested. One sample of tumour tissue from group $\mathrm{B}$ was contaminated during preparation for ICP-MS.

ICP-MS analysis was carried out on a Perkin-Elmer NexION 350X. Internal calibration standard 103-Rh was used. 158-Gd was measured in standard mode with a 1-s integration time. Each sample analysis was run in triplicate reporting the average result with standard deviation.

GLD is an employee of NH TherAguix SAS that is developing the clinical applications of the AGuIX nanoparticles.

\section{Supplementary information}

Supplementary information accompanies this paper at https://doi.org/10.1186/s12645-020-00065-5.

Additional file 1. Section of cine-MRI sequence taken to monitor rats during radiation delivery. Sequence is started before radiation delivery, with beam-on occurring at 50 frames into the movie without degradation in image quality.

\section{Acknowledgements}

The authors thank Natalie Stunnell and Alison Richards of the Ingham Institute's Biological Resources Unit for their support of this project and Dr Nick Proschogo for running the ICP-MS analysis.

\section{Authors' contributions}

HLB: Ran experiments, acquired and analysed data, drafted and edited manuscript. GLD: Substantial contribution to the conception of the work. Manuscript review. FL: Substantial contribution to the conception of the work. Manuscript review. OT: Substantial contribution to the conception of the work. Manuscript review. NMH: Substantial contribution to design of surgical procedure; substantial contribution to carrying out surgical procedures. Manuscript review. AJ: Substantial contribution to experimental design. Substantial contribution to acquiring data. Manuscript review. UJ: Substantial contribution to MRI-linac experiment design and data acquisition. Manuscript review. BD: Substantial contribution to MRI-linac experiment design and data acquisition. Manuscript review. GL: Substantial contribution to MRI-linac experiment design, data acquisition and interpreting results. Manuscript review. TLR: Substantial contribution to experimental design. Substantial contribution to acquiring data, interpreting results and revising manuscript. ZK: Substantial contribution to conception and design of the work, interpreting data and revising manuscript. All authors read and approved the final manuscript.

\section{Funding}

The Australian MRI-linac is supported by funding from the Australian National Health and Medical Research Council Program, grant number: APP1 132471. TLR was supported by a Cancer Institute New South Wales Future Research Leader fellowship and funding from the CINSW funded translational cancer research center, CONCERT. Nanoparticles supplied at reduced cost from $\mathrm{NH}$ TherAguix, France.

\section{Availability of data and materials}

The datasets used and/or analysed during the current study are available from the corresponding author on reasonable request.

\section{Ethics approval and consent to participate}

All animal experiments were approved by the Animal Care and Ethics committee of Western Sydney University (ACEC number A12431) and were conducted in accordance with the "Australian Code for the Care and Use of Animals for Scientific Purposes (2013)" and the "New South Wales Animal Research Act 1985". 


\section{Consent for publication}

Not applicable.

\section{Competing interests}

FL and OT disclose the patent WO2011/135101. GLD and OT disclose the patent WO2009/053644. These patents protect the nanoparticles described in this publication: AGuIX ${ }^{\circledR}$

\section{Author details}

${ }^{1}$ Institute of Medical Physics, School of Physics, The University of Sydney, Sydney, NSW 2006, Australia. ${ }^{2}$ NH TherAguix, 69100 Villeurbanne, France. ${ }^{3}$ Institut Lumière-Matière, Université Claude Bernard Lyon 1, CNRS UMR5306, 69622 Villeurbanne, France. ${ }^{4}$ Institut Universitaire de France (IUF), Paris, France. ${ }^{5}$ School of Psychology, University of New South Wales, Sydney 2052, NSW, Australia. ${ }^{6}$ Ingham Institute for Applied Medical Research, Liverpool, NSW 2170, Australia. ${ }^{7}$ School of Medicine, Western Sydney University, Campbelltown, NSW 2560, Australia.

Received: 2 July 2020 Accepted: 23 September 2020

Published online: 06 October 2020

\section{References}

Acharya S, Fischer-Valuck BW, Kashani R, et al. Online magnetic resonance image guided adaptive radiation therapy: first clinical applications. Int J Radiat Oncol Biol Phys. 2016;94:394-403. https://doi.org/10.1016/j.jijobp.2015.10.015.

Bobyk L, Edouard M, Deman P, et al. Photoactivation of gold nanoparticles for glioma treatment. Nanomed Nanotechnol Biol Med. 2013;9:1089-97. https://doi.org/10.1016/j.nano.2013.04.007.

Brown JMC, Hanna GG, Lampe N, et al. Towards photon radiotherapy treatment planning with high Z nanoparticle radiosensitisation agents: The Relative Biological Effective Dose (RBED) framework. Cancer Nanotechnol. 2018. https ://doi.org/10.1186/s12645-018-0043-7.

Butterworth KT, McMahon SJ, Currell FJ, Prise KM. Physical basis and biological mechanisms of gold nanoparticle radiosensitization. Nanoscale. 2012;4:4830-8. https://doi.org/10.1039/c2nr31227a.

Byrne H, McNamara A, Kuncic Z. Impact of nanoparticle clustering on dose radio-enhancement. Radiat Prot Dosimetry. 2019. https://doi.org/10.1093/rpd/ncy218.

Byrne H, Gholami Y, Kuncic Z. Magnetic nanoparticles for MRI and radiotherapy. Phys Med Biol Roadmap Nanopart Radiat Ther. 2020.

Center for Drug Evaluation and Research (2019) FDA Drug Safety Communication: FDA warns that gadolinium-based contrast agents (GBCAs) are retained in the body; requires new class warnings. FDA.

ClinicalTrials.gov Radiosensitization of Multiple Brain Metastases Using AGuIX Gadolinium Based Nanoparticles. In: ClinicalTrials.gov.https://clinicaltrials.gov/ct2/show/NCT02820454. Accessed 16 June 2020a.

ClinicalTrials.gov Radiotherapy of Multiple Brain Metastases Using AGuIX ${ }^{\circledR}$. In: ClinicalTrials.gov.https://clinicaltrials.gov/ ct2/show/NCT03818386. Accessed 16 June 2020b.

Corradini S, Alongi F, Andratschke N, et al. MR-guidance in clinical reality: current treatment challenges and future perspectives. Radiat Oncol. 2019. https://doi.org/10.1186/s13014-019-1308-y.

Detappe A, Kunjachan S, Rottmann J, et al. AGuIX nanoparticles as a promising platform for image-guided radiation therapy. Cancer Nanotechnol. 2015. https://doi.org/10.1186/s12645-015-0012-3.

Dufort S, Bianchi A, Henry M, et al. Nebulized gadolinium-based nanoparticles: a theranostic approach for lung tumor imaging and radiosensitization. Small. 2015:11:215-21. https://doi.org/10.1002/smll.201401284.

Fallone BG. The rotating biplanar linac-magnetic resonance imaging system. Semin Radiat Oncol. 2014;24:200-2. https:// doi.org/10.1016/.jsemradonc.2014.02.011.

Hainfeld JF, Smilowitz HM, O'Connor MJ, et al. Gold nanoparticle imaging and radiotherapy of brain tumors in mice. Nanomedicine. 2013:8:1601

Keall PJ, Barton M, Crozier S. The Australian Magnetic Resonance Imaging-Linac Program. Semin Radiat Oncol. 2014;24:203-6. https://doi.org/10.1016/j.semradonc.2014.02.015.

Kiviniemi A, Gardberg M, Ek P, et al. Gadolinium retention in gliomas and adjacent normal brain tissue: association with tumor contrast enhancement and linear/macrocyclic agents. Neuroradiology. 2019;61:535-44. https://doi. org/10.1007/s00234-019-02172-6

Klüter S. Technical design and concept of a 0.35 T MR-Linac. Clin Transl Radiat Oncol. 2019;18:98-101. https://doi. org/10.1016/j.ctro.2019.04.007.

Kuncic Z, Lacombe S. Nanoparticle radio-enhancement: principles, progress and application to cancer treatment. Phys Med Biol. 2018;63:02TR01. https://doi.org/10.1088/1361-6560/aa99ce.

Le Duc G, Miladi I, Alric C, et al. Toward an image-guided microbeam radiation therapy using gadolinium-based nanoparticles. ACS Nano. 2011:5:9566-74. https://doi.org/10.1021/nn202797h.

Lechtman E, Mashouf S, Chattopadhyay N, et al. A Monte Carlo-based model of gold nanoparticle radiosensitization accounting for increased radiobiological effectiveness. Phys Med Biol. 2013;58:3075. https://doi. org/10.1088/0031-9155/58/10/3075.

Liney GP, Dong B, Weber E, et al. Imaging performance of a dedicated radiation transparent RF coil on a 1.0 Tesla inline MRI-linac. Phys Med Biol. 2018a;63:135005. https://doi.org/10.1088/1361-6560/aac813.

Liney GP, Jelen U, Byrne H, et al. Technical Note: The first live treatment on a 1.0 Tesla inline MRI-linac. Med Phys. 2019. https://doi.org/10.1002/mp.13556.

Liney GP, Whelan B, Oborn B, et al. MRI-linear accelerator radiotherapy systems. Clin Oncol. 2018b. https://doi. org/10.1016/j.clon.2018.08.003.

Lux F, Tran VL, Thomas E, et al. AGulX ${ }^{\circledR}$ from bench to bedside-transfer of an ultrasmall theranostic gadolinium-based nanoparticle to clinical medicine. BJR. 2018;92:20180365. https://doi.org/10.1259/bjr.20180365. 
McMahon SJ, Hyland WB, Brun E, et al. Energy dependence of gold nanoparticle radiosensitization in plasmid DNA. J Phys Chem C. 2011;115:20160-7. https://doi.org/10.1021/jp206854s.

McNamara AL, Kam WWY, Scales N, et al. Dose enhancement effects to the nucleus and mitochondria from gold nanoparticles in the cytosol. Phys Med Biol. 2016;61:5993-6010. https://doi.org/10.1088/0031-9155/61/16/5993.

Mignot A, Truillet C, Lux F, et al. A top-down synthesis route to ultrasmall multifunctional Gd-based silica nanoparticles for theranostic applications. Chemistry. 2013;19:6122-36. https://doi.org/10.1002/chem.201203003.

Porcel E, Li S, Usami N, et al. Nano-Sensitization under gamma rays and fast ion radiation. J Phys: Conf Ser. 2012;373:012006. https://doi.org/10.1088/1742-6596/373/1/012006.

Raaymakers BW, Jürgenliemk-Schulz IM, Bol GH, et al. First patients treated with a 1.5 T MRI-Linac: clinical proof of concept of a high-precision, high-field MRI guided radiotherapy treatment. Phys Med Biol. 2017;62:L41-50. https://doi. org/10.1088/1361-6560/aa9517.

Schmidek HH, Nielsen SL, Schiller AL, Messer J. Morphological studies of rat brain tumors induced by N-nitrosomethylurea. J Neurosurg. 1971;34:335-40. https://doi.org/10.3171/jns.1971.34.3.0335.

Verry C, Dufort S, Barbier EL, et al. MRI-guided clinical 6-MV radiosensitization of glioma using a unique gadolinium-based nanoparticles injection. Nanomedicine. 2016;11:2405-17. https://doi.org/10.2217/nnm-2016-0203.

Verry C, Dufort S, Lemasson B, et al. Targeting brain metastases with ultrasmall theranostic nanoparticles, a first-in-human trial from an MRI perspective. Sci Adv. 2020;6:eaay5279. https://doi.org/10.1126/sciadv.aay5279.

Verry C, Sancey L, Dufort S, et al. Treatment of multiple brain metastases using gadolinium nanoparticles and radiotherapy: NANO-RAD, a phase I study protocol. BMJ Open. 2019;9:e023591. https://doi.org/10.1136/bmjopen-2018023591.

Winkel D, Bol GH, Kroon PS, et al. Adaptive radiotherapy: The Elekta Unity MR-linac concept. Clin TransI Radiat Oncol. 2019;18:54-9. https://doi.org/10.1016/j.ctro.2019.04.001.

\section{Publisher's Note}

Springer Nature remains neutral with regard to jurisdictional claims in published maps and institutional affiliations.

- fast, convenient online submission

- thorough peer review by experienced researchers in your field

- rapid publication on acceptance

- support for research data, including large and complex data types

- gold Open Access which fosters wider collaboration and increased citations

- maximum visibility for your research: over 100M website views per year

At BMC, research is always in progress.

Learn more biomedcentral.com/submissions 